\title{
An exploratory investigation of the use and effects of academic instant messaging groups among university students
}

\author{
Daniel B. le Roux ${ }^{1}$ (D) . Douglas A. Parry ${ }^{1}$ (i)
}

Received: 12 February 2021 / Accepted: 7 June 2021 / Published online: 7 July 2021

(C) The Author(s), under exclusive licence to Springer Science+Business Media, LLC, part of Springer Nature 2021

\begin{abstract}
The use of instant messaging groups for various academic purposes is a rising, but largely understudied, trend in higher education institutions. In the present study we investigate the use purposes and outcomes of three types of academic instant messaging groups or AIMGs. Formal AIMGs are created and managed by teaching staff, class AIMGs are created by students and joined by all members of a particular class, and study AIMGs are smaller groups created by students that know each other personally or collaborate in group assignments. To advance understanding of the role of these groups in students' wellbeing and academic development, we pose research questions concerning their associations with academic performance, academic stress, and students' course experiences. We adopt an exploratory frame and survey methodology to collect data from a large sample of undergraduate students $(n=1752)$. Our findings indicate that, at the institution where data were collected, high rates of AIMG participation is the norm, with class AIMGs emerging as particularly popular. We find statistically significant interaction between formal and study AIMGs and academic performance, as well as between study AIMGs and academic stress. Participation in these groups also predicts students' social experience of a learning community, as well as their perception of the teaching they receive. Throughout, however, the observed effects are small and their practical significance is questioned.
\end{abstract}

Keywords Academic instant messaging groups - Academic performance Academic stress · Teaching effectiveness $\cdot$ Learning community $\cdot$ WhatsApp

Daniel B. le Roux

dbleroux@sun.ac.za

1 Department of Information Science, Stellenbosch University, Stellenbosch, South Africa 


\section{Introduction}

High levels of smartphone use are common among university students in both the developed and developing worlds (Panova et al., 2020; le Roux et al., 2021). The affordances associated with these media have cultivated a mode of living characterised by permanent online connectedness - a phenomenon which has rapidly emerged over the past decade and of which the individual and social consequences are not yet well understood (Vorderer et al., 2017). A central theme in this domain has concerned the role of social media and their diverse impacts_-positive and negativeon behaviour and well-being (Krause et al., 2019; Orben, 2020; le Roux \& Parry, 2020). Not surprisingly, among university students, social media have also become an important medium for communication and collaboration in relation to academic activities, with instant messaging (IM) applications, in particular, playing a key role in these exchanges (Kim et al., 2014).

In the present study we explore the use of instant messaging among university students with particular emphasis on the formation, adoption, use and effects of multi-user IM groups created for academic purposes. We refer to such groups as academic instant messaging groups (AIMGs). Within this research area, studies can broadly be categorised based on the type of instant messaging group under investigation, with two primary categories emerging. The first category, which includes a majority of studies conducted, concerns AIMGs that are initiated and administered by academic staff (i.e, lecturers, instructors, tutors etc.) as part of a teaching and learning strategy (e.g., Kim et al. 2014; Bouhnik \& Deshen 2014; Willemse 2015; Zulkanain et al. 2020). For the purpose of the present study we refer to these as formal AIMGs. The second category includes only a small collection of studies and concerns AIMGs that are initiated and administered by students for academic purposes, without any involvement of, or participation by, academic staff (e.g., Muktar et al. 2020; Udenze \& Oshionebo 2020; Alghamdi et al. 2016). We refer to these groups as organic AIMGs.

Across studies of both formal and organic AIMGs, researchers have noted that IM platforms offer a wide range of potential advantages over other media used for communication and collaboration in teaching and learning contexts. Some studies have also reported enhanced academic performance when students participate in formal AIMGs over traditional, face-to-face interactions (Barhoumi, 2015; Altaany, 2015; Nitza \& Roman, 2016). However, researchers have also warned that AIMGs may, under certain conditions, introduce risks - both for the individual participant, and for the academic integrity of the course or programme (Bouhnik \& Deshen, 2014; Willemse, 2015; Román et al., 2018).

To contribute to this body of knowledge, we conducted a survey-based, exploratory study targeting a population of undergraduate university students. In addition to investigating the adoption of, motivation for and perceptions about the use of both formal and organic AIMGs, we investigated the relationship between participation in the different group types and academic performance, academic stress, students' perceptions of the quality of their academic programmes and the teaching they receive, as well as their sense of belonging in an academic community. 


\section{Literature review}

\subsection{Theoretical points of departure}

As a theoretical point of departure for the present investigation we adopt the primary premises of Uses and Gratifications Theory $(U \& G)$ which has been widely used in research on the adoption and use of communication media. U\&G posits that individuals seek out media among competitors to fulfil their needs and obtain gratifications (Whiting \& Williams, 2013). This contrasts U\&G from earlier theories of communication which adopted a more passive view of media consumers - "the audience is characterised as active, discerning, and motivated in their media use" (p. 351 Quan-Haase \& Young 2010). For example, findings by Leung (2001) indicate that instant messaging is used to fulfil a range of needs including showing affection, being entertained, having fun, killing time, relaxing, and escaping from pressures and responsibilities.

We propose, accordingly, that the adoption and use of AIMGs are driven by particular needs among students and, based on the review of extant literature on AIMGs, we developed an initial list of such needs to structure our empirical investigation.

However, while U\&G provides a meaningful theoretical lens for the study of the motivations and purposes of AIMG use, it does not explicitly speak to the potential outcomes of such use for academic performance, anxiety or course experiences. Acknowledging the recent emergence of AIMGs in general and organic AIMGs in particular, we adopted an exploratory frame and based our research questions on recently reported empirical data of the associations between these constructs. Moreover, the three dependent variables of interest to us differ to the extent that a single theoretical framework would be inadequate. Accordingly, where relevant, we note theories which potentially describe these relationships.

In the sections which follow we provide a concise review of relevant literature. The first section addresses studies of formal AIMGs, while the second addresses organic AIMGs. Thereafter, we briefly outline extant findings in relation to the association between participation in AIMGs, academic performance, academic stress and students' experiences of their academic programmes.

\subsection{Formal AIMGs}

Effective and efficient communication among students and academic staff is an important dimension of any teaching and learning strategy in higher education. The potential of smartphones and IM applications to enhance such communication has received attention in a wide range of disciplines, with early studies appearing more than a decade ago (Hickerson \& Giglio, 2009). Across these studies a common theme is the adoption of experimental or action research approaches to compare various aspects of student behaviour and/or performance based on their participation or non-participation in a formal AIMG as part of a course. The studies are motivated by a range of perceived benefits associated with the affordances offered by the 
medium. These include, amongst others, low cost, ease of use, mobility and multimedia support (Hickerson \& Giglio, 2009; Barhoumi, 2015; Bouhnik \& Deshen, 2014; Willemse, 2015; Suardika et al., 2020).

A key advantage of AIMGs over email, which is often used for student-lecturer interaction, is the increased immediacy afforded by the reduction in the technical friction associated with interaction. Poblete and Nieto (2020) compare the reception and response time of a group of students using group email $(n=32)$ with a group using a WhatsApp-based AIMG $(n=27)$. WhatsApp is a free, mobile-centric IM platform used by more than two billion people in over 180 countries across the world (WhatsApp, 2020). Results indicated that communication in the AIMG was significantly faster in terms of both reception and response time (Poblete \& Nieto, 2020). Accordingly, formal AIMGs are associated with an increased volume of student-instructor interactions (Hickerson \& Giglio, 2009).

Researchers have also investigated the potential of formal AIMGs to encourage and enhance collaborative learning among students (e.g., Barhoumi 2015; Nitza \& Roman 2016; Ntinda \& Bidwell 2018; Basitere et al. 2019; Udenze \& Oshionebo 2020). Across these studies results indicate that students are both willing and able to utilise formal AIMGs to share knowledge and engage in collaborative problem solving, particularly when they enjoy some degree of anonymity. Willemse (2015, p. 5), for instance, report that anonymous participation in AIMGs encourages engagement due to the reduction of fear of criticism from peers.

Participation in formal AIMGs has also been associated with a stronger sense of community among students (Suardika et al., 2020), increased subject interest (Román et al., 2018), improved performance in a number of competencies (including teamwork, adaptability and continuous learning; Pérez-Jorge et al. 2018), and improved performance in the learning of a foreign language (Avci \& Adiguzel, 2017).

Notwithstanding the positive aspects of formal AIMGs, studies have also identified barriers to and potential risks of their adoption. Prominent among these is the rise in staff workload that results from the increased volume of interactions and the expectation to be available for interaction at all times (Bouhnik \& Deshen, 2014; Zulkanain et al., 2020). The high volume of messages from both staff and students also creates an increased possibility of the distribution of false information, confusion and misinterpretations of statements (Willemse, 2015; Zulkanain et al., 2020; Udenze \& Oshionebo, 2020). In contexts where not all students enjoy equal access to devices and Internet connectivity, a formal AIMG may lead to information asymmetry (Willemse, 2015). Researchers have also warned that many students may struggle to learn effectively from participation in AIMGs (Zulkanain et al., 2020; Rahmadi, 2020), particularly when their personal or social use of the IM platform continuously distracts them from their academic use thereof (Zulkanain et al., 2020).

\subsection{Organic AIMGs}

While formal AIMGs are initiated and administered by academic staff members, studies have shown that university students also create their own IM groups for academic purposes (Muktar et al., 2020; Alghamdi et al., 2016; Fuentes Gutiérrez et al., 2017). These organic AIMGs have received very little research attention and various 
aspects of their operation, use patterns and effects remain unknown. Nonetheless, as argued by Fuentes Gutiérrez et al. (2017), they play an increasingly important role in shaping the dynamics of interpersonal and inter-group interaction among university students.

Alghamdi et al. (2016), based on a study of "unmonitored, self-created" WhatsApp groups among students undertaking distance learning, report that the medium offers students various benefits. These include collaboration with peers, the sharing of administrative information like important dates and deadlines, and support in their efforts to make sense of study material. As is the case with formal AIMGs, the ease of use of IMGs was found to be a key motivator for its adoption. Muktar et al. (2020) investigate the use of WhatsApp among undergraduate students through the lens of self-regulated learning theory. The authors find that the medium plays an important role in "scaffolding" learning processes by providing students with immediate and easy access to vital information, as well as facilitating academic interaction and social development. Fuentes Gutiérrez et al. (2017) utilise a discussion group methodology to investigate the use of organic WhatsApp groups in the university classroom context. Supported by Muktar et al. (2020), the authors conclude that the potential of the medium is mainly a product of the immediacy it offers, coupled with its plasticity and capacity to generate personal ties.

While acknowledging these benefits, Basitere and Ivala (2017) find evidence that the use of organic AIMGs enables, what they refer to as, 'cheating collaboration'. This occurs when the affordances of the medium enable students to share answers or solutions to tests and assignments, creating the possibility of shortcuts to achieving academic outcomes. Moreover, the absence of the lecturer increases the possibility of a high volume of irrelevant messages (Udenze \& Oshionebo, 2020), as well as the dissemination of false or inaccurate information (Muktar et al., 2020).

\subsection{Comparison of formal and organic AIMG content}

In a comparative analysis of content in formal and organic AIMGs, Baishya and Maheshwari (2020) found that messages in these groups can be classified according to six broad themes: regular academics, exam and evaluation, extracurricular activities, entertainment, wishes and greetings, and general discussion of social or non-academic topics. Their analysis indicates, firstly, that the volume of messages was substantially lower in formal groups - 123 messages in the formal versus 1404 messages in the organic AIMG - during the month of data collection. Secondly, they found that discussions in the formal group adopt a more formal conversational style, with messages primarily concerning academic matters. Finally, Baishya \& Maheshwari (2020, p. 36) found that almost a third of the messages in the organic AIMG concerned entertainment ("sharing or linking of photos and videos for the main purpose of fun").

Given the relative dearth of prior research about the use patterns, purposes and motivations of formal and organic AIMGs, we posed the following two research questions.

RQ1: How common is participation in formal and organic AIMGs among undergraduate university students? 
RQ2: What are the purposes and motivations for use of formal and organic AIMGs?

\subsection{AIMGs and academic performance}

The small number of studies that have investigated the impact of formal AIMG participation on academic performance typically rely on small sample sizes and ad hoc, unvalidated measures of performance in particular assessments. Barhoumi (2015), for example, found improved performance in test scores for students who engaged in a weekly, hour-long IM discussion about course content $(n=34)$, compared to those who performed the activity face-to-face $(n=34)$. Avci and Adiguzel (2017) investigate the use of WhatsApp groups for university students' learning of a foreign language. The authors report that participation in such groups was associated with increased performance and improved work quality. Nitza and Roman (2016) show a significant positive relationship between the achievements of WhatsApp users during a case study seminar course $(n=120)$ and their satisfaction with the course $(r=0.819)$. However, the relationship between WhatsApp group participation and academic performance is not reported.

Despite the various shortcomings in these studies, the totality of evidence suggests that the utilisation of formal AIMGs has the potential to enhance the performance of participating students. However, as argued by Zulkanain et al. (2020), this outcome depends upon the enactment of the medium such that it effectively supports learning processes and requires active participation of lecturers to align the content of conversations with learning outcomes.

There is also a general absence of studies that investigate the association between academic performance and organic AIMG participation. However, a number of studies have considered the use of social media for academic purposes in relation to students' performance. In their study of 400 medical students in Saudi Arabia, AlFaris et al. (2018) find that $36 \%$ of students use WhatsApp for learning purposes, but find no association between such use and academic performance. Afful and Akrong (2020), in a study among students in Ghana $(n=295)$, find a small, albeit not statistically significant, positive association between self-reported time spent on WhatsApp for academic purposes and academic performance measured as cumulative GPA ( $\beta=0.095, p<0.1)$. Al-Rahmi and Zeki (2017) find that social media use for learning is positively associated with both collaborative learning and student satisfaction. These two factors, in turn, were positively associated with academic performance.

It may be argued that, much like formal AIMGs, organic AIMGs have the potential to scaffold the learning process by providing students with immediate access to their peers and, through them, access to administrative information, learning material and knowledge sharing. However, unlike formal AIMGs where lecturers can moderate conversation and correct false information, organic AIMGs can potentially disrupt learning through high volumes of irrelevant messages and inaccurate information.

Considering their increasingly important role in student interaction and collaboration (Fuentes Gutiérrez et al., 2017), their association with academic performance is of interest. Accordingly, we posed the following research question: 
RQ3: What is the nature of the association between participation in AIMGs and self-reported academic performance?

\subsection{AIMGs and academic stress}

Due to a range of factors, including developmental and social changes, financial and accommodation problems, as well as work and academic demands, university students experience "significantly greater than average levels of stress" (Lakaev, 2009, p. 56). Macgeorge et al. (2005, p. 365), accordingly, argue that, while university is a positive experience for many students, "many students also experience college as chronically stressful due to academic requirements" like tests, papers and presentations.

The role of formal and/or organic AIMGs in student stress has not received research attention. However, Mogashana et al. (2019) investigate the potential of WhatsApp groups specifically designed to provide students with psychosocial support through a small group life coaching programme. Results indicated that immediacy of interactions through the WhatsApp group served to bridge physical separation between the coach and students, fostering ongoing communication and support. This aligns with well-documented evidence that the perceived availability of support of a network of caring and accessible members influences health outcomes after stressful events (Macgeorge et al., 2005).

While AIMGs are not necessarily initiated with the aim of psychosocial support, Kim et al. (2014, p. 39), in a study of AIMG use among Korean students, find that, under certain circumstances, AIMGs offer a friendly environment where students feel comfortable to share personal matters "using various emotional and social expressions." Zulkanain et al. (2020), accordingly, argue that the expression of emotions or experiences of problems unrelated to class, serve to cultivate stronger relationships among group members. We propose, accordingly, that AIMGs may, under certain circumstances, serve to alleviate academic stress by providing students with a network of supportive peers and enabling expressions of care and encouragement.

We are mindful, however, of the possibility that AIMGs may also aggravate academic stress. We foresee two potential mechanisms for this effect. Firstly, studies have suggested that the high volume of messages associated with organic AIMGs can create experiences of information overload (Baishya \& Maheshwari, 2020), as well as distract students during academic activities (Zulkanain et al., 2020). Such media-based distractions have been associated with the procrastination of academic tasks and increased academic stress (le Roux \& Parry, 2021). Secondly, based on the general premises of social contagion, we propose that messages in which students express their own concern, fear or anxiety about upcoming tests or exams may evoke or worsen experiences academic stress among their peers. Social contagion refers to the "involuntary catching of behaviours and attitudes across connected individuals" (Burgess et al., 2018, p. 164). In extant research on the role of social contagion in educational contexts, evidence indicates that "student attitudes, beliefs, values and behaviours are influenced by natural peer contexts" (Parr \& Townsend, 2002, p. 409 ), and that this may include levels of motivation and perceptions of academic self-efficacy (Burgess et al., 2018). 
Given the lack of evidence on the interaction between formal and organic AIMG use and academic stress, we posed the following research question:

RQ4: What is the nature of the association between participation in AIMGs and academic stress?

\subsection{AIMGs and course experience}

In addition to academic performance and stress, we propose, based on extent literature, that AIMG participation has the potential to impact two dimensions of students' perceptions about their academic programmes. Firstly, due to their ability to increase the immediacy, quality and quantity of student-lecturer interactions (Hickerson \& Giglio, 2009; Poblete \& Nieto, 2020; Willemse, 2015), formal AIMGs are likely to influence students' perceptions of the quality of the teaching they receive. Román et al. (2018) report that a majority of students believe that IM-based interaction with their lecturers would increase their motivation and interest, and that $90 \%$ of students believe such interaction is appropriate. While these findings suggest that formal AIMGs would be positively associated with perceptions of teaching quality, it should be acknowledged that the potential negative consequences of poorly managed formal AIMGs (see Section 2.2) may lead to negative experiences and perceptions of teaching quality.

The role of organic AIMGs in perceptions of teaching quality has not been investigated. We propose, however, that the interactions in these groups will, at times, involve students' views of their lecturers and the quality of teaching they receive. In accordance with Fuentes Gutiérrez et al. (2017) we argue that interactions on organic AIMGs will impact the dynamics of relations between students and their teaching staff and, as part thereof, perceptions about the abilities of and effort made by teaching staff.

The second dimension of course experience which, based on earlier studies, may be influenced by AIMGs is students' sense of being part of a learning community. Suardika et al. (2020), through a comparative study of students attending conventional face-to-face classroom instruction $(n=51)$ and students using WhatsApp for learning $(n=49)$, find that the WhatsApp group reported a stronger sense of community. This likely results from increased levels of interaction and the sharing of personal information (Kim et al., 2014), as well as knowledge sharing, collaborative learning, or problem solving (Nitza \& Roman, 2016; Barhoumi, 2015; Willemse, 2015) - factors which serve to generate strong interpersonal ties (Fuentes Gutiérrez et al., 2017). Based on these factors, it is expected that both formal and organic AIMGs have the capacity to increase students' sense of being part of an academic community. However, as Román et al. (2018) warn, students may experience embarrassment or rejection when peers ridicule, ignore or reject their ideas during AIMG interactions, and that harassment or cyberbullying can occur in these groups. Such events may have a negative impact on students' sense of belonging to learning community.

To advance knowledge of the interaction between AIMG participation and students' experiences of their degree programmes, we posed the following two research questions: 
RQ5: What is the nature of the association between participation in AIMGs and students' perception of the quality of teaching they receive?

RQ6: What is the nature of the association between participation in AIMGs and students' social experience of belonging to a learning community?

\section{Method}

To address our research questions, we adopted a survey methodology and conducted an exploratory study with a sample of undergraduate university students. In accordance with recent calls for greater transparency in our research processes (e.g., Bowman and Keene 2018; Dienlin et al. 2020; Lewis 2020), following IRB approval, given the need for clarity around the timing of analytic decisions, we specified a pre-analysis plan prior to data collection (available at: https://osf.io/pd42h/). Any deviations from our pre-analysis plan and any further exploratory analyses will be explicitly labelled as such. Additionally, all materials, data, and analysis scripts are also available through the OSF.

\subsection{Participants and procedure}

We targeted an undergraduate student sample at a large residential university in South Africa. After development of an initial version of our survey, we performed a pilot test with a small group of students $(n=7)$ in their fourth year of study, followed by a focus group discussion to gather feedback. Based on feedback about the use of AIMGs at the institution, we made two important changes to the survey. Firstly, the students stated that, with only rare exceptions, WhatsApp is the platform used for AIMGs at the institution and, to ensure clarity, we updated the survey to refer to "WhatsApp groups for academic purposes" rather than "academic instant messaging groups." While this phrasing would reduce ambiguity in our survey items, we are mindful that our data do not reflect the use of non-Whatsapp AIMGs at the institution. Secondly, the focus group participants made a strong distinction between two forms of organic AIMGs. The first, which we refer to as study AIMGs, are groups involving a small number of students (typically less than 10) from a class. Members of a study AIMG generally know each other on a personal level and/or work together on group assignments. The second form of organic AIMG, which we refer to as class AIMGs, are created to include all students in a particular class. The group is usually created by the student representative and students are added to the group by providing him/her with their phone numbers. We updated our AIMG typology accordingly, a summary of which is presented in Table 1.

To recruit respondents, after receiving IRB approval, email invitations to complete the final version of the survey on an online survey platform (Checkbox) were sent to 20196 undergraduate students at the institution in November 2020. Importantly, we collected data approximately eight months after the institution switched from faceto-face to online teaching due to the closure of its campuses in response to Covid-19. While we believed that the dominant norms and practices around AIMG creation 
Table 1 Typology of academic instant messaging groups

\begin{tabular}{lll}
\hline AIMG Type & Created and administered by & Membership \\
\hline Formal & $\begin{array}{l}\text { Academic staff (e.g., lecturer or } \\
\text { tutor) }\end{array}$ & $\begin{array}{l}\text { Lecturer/tutor and all members of class or } \\
\text { tutorial group }\end{array}$ \\
Class & Students & $\begin{array}{l}\text { Open to all members of a class } \\
\text { Study }\end{array}$ \\
Students & $\begin{array}{l}\text { Selected members of a class that know each } \\
\text { other personally or collaborate in group } \\
\text { assignments }\end{array}$ \\
\hline
\end{tabular}

and use would continue to determine their functioning, we expected that the switch to online learning would lead to some changes. However, the absence of baseline data for comparison implied that we would be unable to identify or describe these changes meaningfully. Accepting these limitations, we endeavoured to interpret our data with attention to the potential role that online teaching may have played in AIMG functioning.

To detect a minimally interesting effect size of $R^{2}=0.05$ (a small effect size given conventional benchmarks) for a multiple regression analysis with alpha $=0.05$, $80 \%$ power, the AIMG participation variables as predictors, and controlling for the demographic variables and the remaining variables of interest, we determined that we required a minimum sample of at least 430 completed responses to the survey. The survey remained open for a period of 14 days and, in this time, it was accessed 2990 times. After removing 1220 incomplete responses and 18 responses for which consent was not provided, our final sample of $n=1752$ was established. The sample included more female $(63.3 \%)$ than male $(36.2 \%)$ respondents, with only $0.5 \%$ using other gender descriptors. The mean age of respondents is 21.12 years $(\mathrm{SD}=$ 2.81 ), and $26.9 \%$ are in their first year of study, $28.1 \%$ their second, $28 \%$ their third, and $17 \%$ have been enrolled for four or more years. Respondents represented 10 of the academic faculties at the institution, with 339 from Economic and Management Sciences (19.3\%), 324 from Engineering (18.5\%), 278 from Arts and Social Sciences (15.9\%), 271 from Medicine and Health Sciences (15.5\%), 228 from Science (13\%) and the remainder from Agriculture (6.6\%), Law (5.6\%), Education (4.2\%), Theology (1.1\%) and Military Science $(0.3 \%)$.

\subsection{Measures}

To produce relevant data to address our research questions we used a combination of existing scales and, where necessary, developed new measures based on the literature reviewed. The complete survey is available through the OSF (https://osf.io/qpva7/).

To describe the demographics of our sample, respondents were asked to indicate the gender they identify as (male, female, or open), the faculty within which their academic program resides, their year of birth, and their current year of study. Next, to assess self-reported overall academic performance we used a single item that asked respondents to indicate their average academic performance in their courses or 
modules. Response options ranged from less than $40 \%$ to more than $85 \%$, with $5 \%$ intervals.

To quantify AIMG participation we considered two metrics: the proportion of a respondents' modules (or courses) in which a group type was used and the frequency with which a respondent reported engaging in three group-level behaviours. To determine the proportion of the respondents' modules in which they participate in a particular type of AIMG we presented three items, one for each group type. These items required respondents to indicate, using a five-point Likert scale with response options ranging from zero ("In none of my modules") to four ("In all of my modules"), the proportion of their modules in which they participated in 1) "Whatsapp groups created by the lecturer or tutor"; 2) "Whatsapp groups created by students for everyone in the class to join"; and 3) "Whatsapp groups for a smaller group of classmates, like a study group or project team." If a respondent indicated that they did not participate in a particular type of AIMG, no further items for that group type were presented to them, with their responses coded as zero to indicate non-participation. For participants who indicated at least some level of participation in a group type we presented three sets of items concerning their participation frequency, participation purpose, and the perceived outcomes of participation for each of the three group types.

For participation frequency, for each type of group, three items were presented. These items concerned the frequency with which respondents 1) read messages, 2) posted messages, and 3) shared files in an AIMG. Responses were recorded using five-point likert scales with values as follows: "never" (0); "a couple of times per semester" (0.25); "a couple of times per week" (0.5); "daily" (1); and "multiple times per day" (2). For each respondent and each group type, responses to these three items were summed to produce frequency scores with a possible range of 0 to 6 . To produce an overall participation score for each group type we calculated the product of participation proportion and participation frequency. This resulted in three scales (one for each group type) that, with a range of 0 to 24 , provide an indication of the respondents' self-reported level of participation in AIMGs on Whatsapp.

To assess the purposes for which each of the group types were used we presented seven items, separately for each type of AIMG. These items concerned whether respondents use these groups to 1) communicate administrative information like class or test dates and times; 2) discuss academic content covered in the module; 3 ) share class notes or other academic resources; 4) share answers or solutions for assignments; 5) coordinate group work; 6) encourage and support each other; and 7) share non-academic content like jokes or memes. Responses could be provided on a fivepoint Likert scale ranging from never to always and were, rather than being summed to form a scale, considered individually as each item represents a distinct use purpose.

To assess respondents' perceptions of the outcomes associated with participation in each type of AIMG we presented, separately, eight statements, informed by the literature reviewed, that represent possible outcomes experienced. These items (available through the OSF) concerned the perceived effect of participation on respondents' access to accurate administrative information, access to useful academic material, understanding of the work, academic performance, stress about their academic work, ability to cope with academic pressure, ability to perform effective group work, and 
anxiety about their work. Respondents indicated their level of agreement for each statement through a five-point agreement scale ranging from "strongly disagree" to "strongly agree". As with use-purposes, responses to these items were not aggregated but were instead considered individually.

Academic stress was assessed using the Lakaev academic stress response scale (LASRS; Lakaev 2009). This scale was developed to quantify stress responses among university students in four stress domains: physiological, behavioural, cognitive, and affective. The scale consists of 21 items, each representing a stress symptom, including: "there is so much going on that I can't think straight", "I felt worried about coping with my studies", and "my work built up so much that I felt like crying." Respondents indicated extent to which they experienced these symptoms on a 5-point Likert scale ranging from "None of the Time" (1) to "All of the Time" (5). Responses to the individual items are summed to form subscale scores and these subscale scores are summed to produce a total LASRS stress response score. A higher score indicates a greater stress response. The LASRS generally has sound psychometric properties (Lakaev, 2009; Javaheri, 2017) and, in the present study, the scale was internally consistent (Cronbach's $\alpha=0.91$ ).

Perceptions of course experience were assessed using two sub-measures from the Australian Course Experience Questionnaire (CEQ): the good teaching scale and the learning community scale (Mcinnis et al., 2001; Griffin et al., 2003). The learning community scale (LCS) includes five items (e.g., " I felt part of a group of students and staff committed to learning") that assess respondents' perceptions of the social experience of learning at university. Responses, provided through a five-point agreement scale ranging from strongly disagree to strongly agree, are aggregated, with higher scores representing a positive perception of the learning community. In the present study, the scale was internally consistent (Cronbach's $\alpha=0.81$ ). The Good Teaching Scale (GTS) assesses various basic elements of effective teaching through five items (e.g., "the teaching staff motivate me to do my best work"), with responses (provided through a five-point agreement scale ranging from strongly disagree to strongly agree) aggregated to form an overall scale. Higher values indicate a perception of better teaching quality. This scale was also found to be internally consistent in the present data (Cronbach's $\alpha=0.85$ ). We also collected responses to the single overall satisfaction item (OSI) to assess respondents' overall satisfaction with their degree programme.

\subsection{Data analysis plan}

All analyses were conducted using the $R$ statistical programming language (v. 4.0.3 R. Core Team 2020), with an alpha level of 0.05 adopted as a threshold for statistical significance. To address our first research question we determined, firstly, the distribution of responses that indicate the proportion of respondents' modules for which each group type is used. Secondly, for each group type, we summarised the frequency with which the three participation forms were reported. Finally, as described in the previous section, overall participation was calculated as the product of proportion and frequency. RQ2 was addressed by providing a descriptive account of the responses for the use purpose and outcome motivation items for each group type. To address our 
four remaining research questions, we first assessed the zero-order bivariate correlations among the overall participation indicators, academic performance, academic stress, the GTS, and the LCS. Following this, we computed separate multiple regression models to determine the predictive capacity of the AIMG participation variables over each of the dependent variables. Each of the regression models controlled for age, gender, faculty, and study-year, as well as all other outcome variables not used as the dependent variable in the model. Deviating from our pre-specified analysis plan, we did not assess gender differences in outcomes, nor did we assess for relationships involving the single overall satisfaction item for education quality.

\section{Findings}

\subsection{Use of academic instant messaging groups}

Of the 1752 respondents, $852(48.63 \%)$ indicated that they did not use formal AIMGs in any of their modules, while $900(51.37 \%)$ used such groups in at least some of their modules and only $164(9.36 \%)$ reported using formal AIMGs in all of their modules. In contrast, for class groups, only $81(4.62 \%)$ respondents reported that they did not use such groups in any of their modules, with the remaining $1671(95.38 \%)$ indicating use of class groups in some modules. At $60.78 \%$ the largest proportion of respondents indicated that they use class AIMGs in all of their modules. For study groups, while $311(17.75 \%)$ respondents reported that they did not use this form of AIMG in any of their modules, of the $1441(82.25 \%)$ respondents who do use such groups, $279(15.82 \%)$ reported that they use these groups in all of their modules. Figure 1 provides a summary of the proportion of respondents' modules in which they reported using the three forms of AIMG. It is evident that, of the three group types, among our sample, use of class groups is the most prevalent across academic modules.

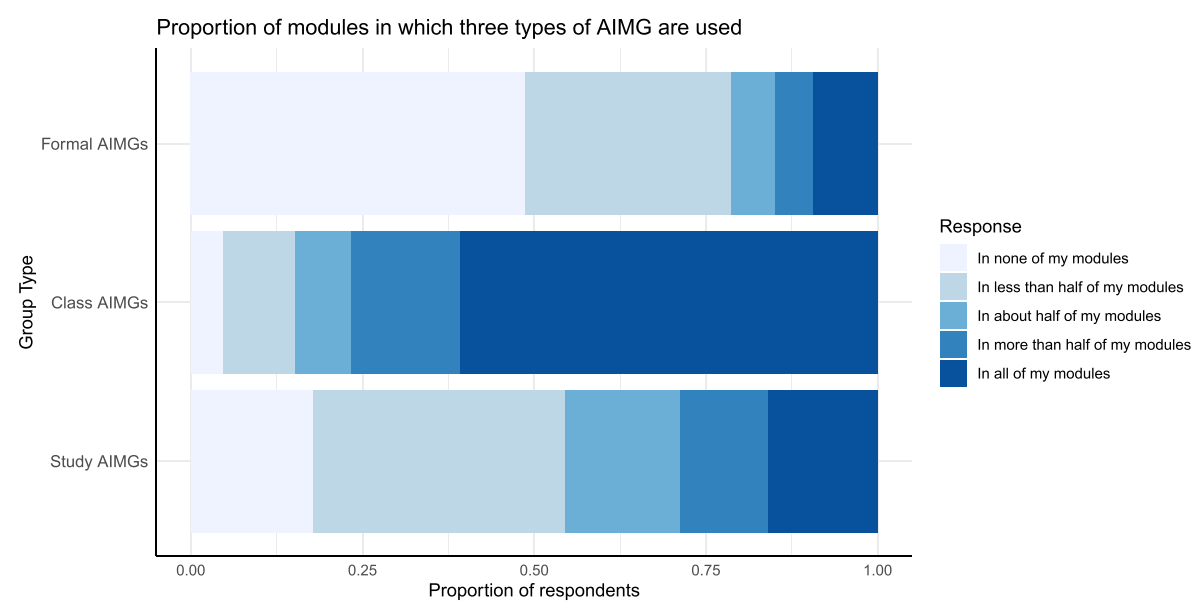

Fig. 1 Proportion of modules in which the three types of AIMGs are used 


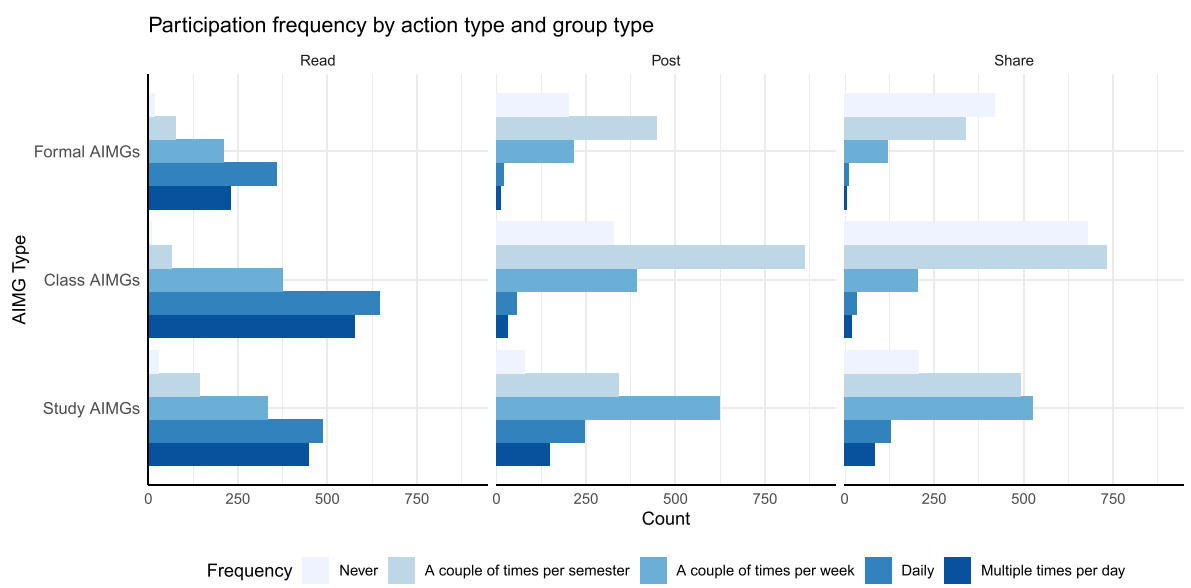

Fig. 2 Participation frequency by action and AIMG type

For respondents who reported using an AIMG type in their modules (formal: $n=900$, class: $n=1671$, study: $n=1441$ ), we considered the frequency with which they reported engaging in three actions: reading, posting, and sharing. For all three group types, as depicted in Fig. 2, participation is characterised more so by reading than by the posting or sharing of content. For formal AIMGs, 591 (65.67\%) respondents reported reading content either daily or multiple times per day, compared to just $34(3.78 \%)$ respondents who reported posting in such groups daily or multiple times per day. Similarly, the number of respondents who reported reading content daily or multiple per day in class $(n=1222,73.13 \%)$ or study groups $(n=936,64.95 \%)$ was larger than the number who indicated that they post in either class $(n=91$, $5.45 \%)$ or study groups $(n=397,27.55 \%)$ daily or multiple times per day. Across action types, with the exception of reading, respondents indicated a greater frequency of involvement in study groups than formal or class groups.

\subsection{Use purposes and outcome perceptions}

Figure 3 provides a visual summary of the purposes for which respondents report using the three types of AIMG. In class and formal groups, the use cases most commonly reported concern the communication of administrative information (formal AIMGs: 40.78\%; class AIMGs: $46.68 \%$ ) and the discussion of course content (formal AIMGs: $36.89 \%$; class AIMGs: $36.74 \%$ ), while for study groups the most frequently reported use case involved the coordination of group work $(45.11 \%)$ followed by the discussion of course content (37.34\%). In all three group types, the two least reported use purposes were the sharing of answers and the sharing of non-academic content. Table 1 in the Supplementary materials provides a detailed description of the response proportions for each use purpose and AIMG type.

Figure 4 visualises the level of agreement with nine perceived outcomes of participating in the three AIMG types. For both formal and class AIMGs, access to accurate administrative information was the outcome that received the most support, while 


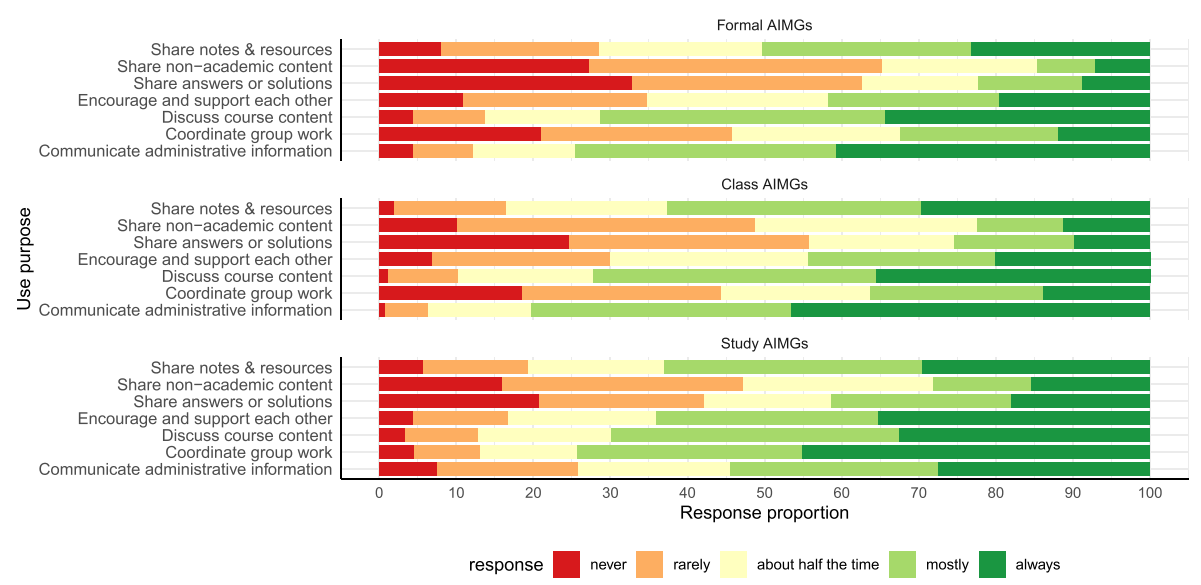

Fig. 3 Use purposes by AIMG type

effective group work was the most reported outcome for study AIMGs. In terms of improving students' academic performance, study AIMGs were perceived to be the most important with $68 \%$ of students either strongly agreeing $(24 \%)$ or agreeing (44\%) that their participation in these groups improves their academic performance. Additionally, 59\% of students either strongly agreed (20\%) or agreed (39\%) that study groups reduce their academic stress and agreed (41\%) or strongly agreed (19\%) that they help them to cope with academic pressure. These findings align with the findings about group use purposes which indicate that students frequently encourage and support each other on study AIMGs, something which occurs less frequently on formal and class AIMGs. Across all three AIMG types, the lowest level of agreement was observed for perceived increases in anxiety about work resulting from participation in AIMGs. Table 2 in the Supplementary materials provides provides a detailed summary of the responses for these items.

\subsection{Relationships between AIMG use and academic performance, academic stress, and perceptions of educational quality}

Table 2 presents the descriptive statistics and bivariate correlations (Spearman's $\rho$ ) for the main study variables. Of the three AIMG types, the highest level of overall participation was found for class AIMGs, followed by study AIMGs. Formal AIMGs, as indicated in Fig. 1 are used much less frequently at the institution. With the exception of the associations between LCS and GTS $(\rho=0.57, p<0.001)$, as well as between study and class AIMG participation $(\rho=0.34, p<0.05)$, all observed correlations are small in magnitude. Positive correlations were found between participation in the three AIMG types. Reported participation in all three group types correlates positively with students' social experience of a learning community (LCS), while formal AIMG participation correlates positively with their perception of effective teaching (GTS) $(\rho=0.13, p<0.001)$. Academic performance is negatively associated with academic stress $(\rho=-0.23, p<0.001)$, but positively associated with both LCS 


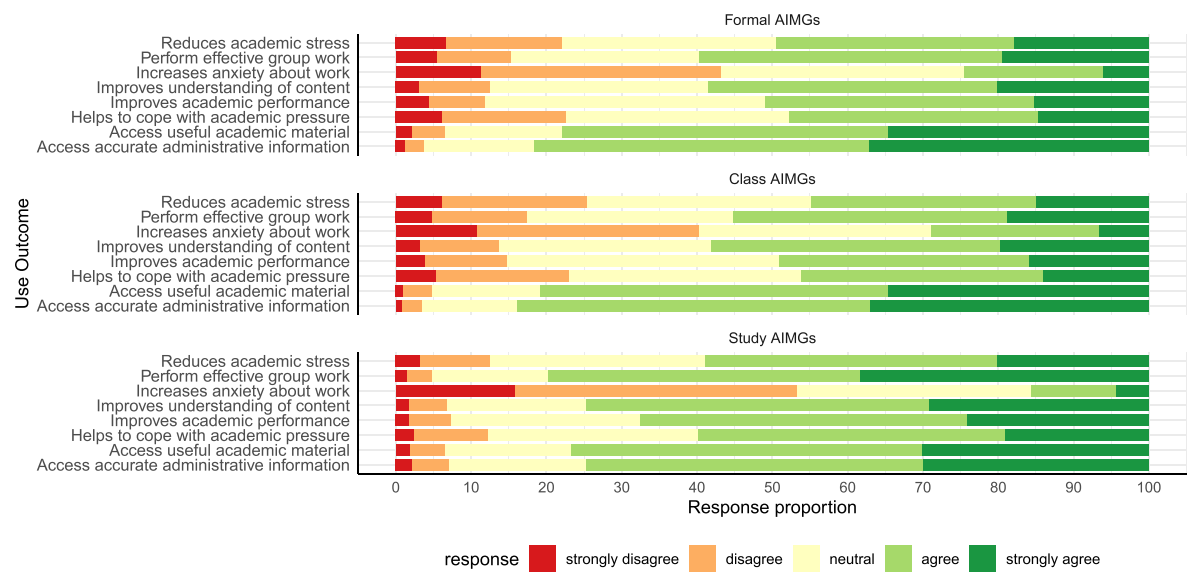

Fig. 4 Use outcomes by AIMG type

$(\rho=0.16, p<0.001)$ and GTS $(\rho=0.06, p<0.05)$. The only correlation found between AIMG participation and academic performance was with participation in study AIMGs $(\rho=0.06, p<0.05)$. Although statistically significant, this association is particularly negligible in magnitude. Academic stress is negatively associated with both GTS $(\rho=-0.27, p<0.001)$ and LCS $(\rho=-0.29, p<0.001)$, but not with participation in any of the three AIMG types.

To address RQ3, we conducted a multiple linear regression predicting academic performance with formal, class and study AIMG participation, academic stress, perception of teaching quality, and social experience of learning community, while controlling for the demographic variables. Table 3 presents the results of this regression. Overall, the model predicts $20 \%$ of the variance in academic performance

Table 2 Descriptive statistics and bivariate correlations for the main study variables

\begin{tabular}{|c|c|c|c|c|c|c|c|c|c|c|}
\hline Variable & Mean & $\mathrm{SD}$ & skewness & kurtosis & 1 & 2 & 3 & 4 & 5 & 6 \\
\hline 1. Formal AIMGs & 1.58 & 2.72 & 2.82 & 10.37 & - & & & & & \\
\hline 2. Class AIMGs & 5.70 & 4.08 & 1.06 & 2.16 & $0.15^{* * *}$ & - & & & & \\
\hline 3. Study AIMGs & 4.22 & 5.04 & 1.92 & 3.83 & $0.08^{* * *}$ & $0.34^{*}$ & - & & & \\
\hline 4. $\mathrm{AP}^{a}$ & 6.64 & 1.8 & 0.07 & -0.35 & 0.02 & 0.06 & $0.06^{*}$ & - & & \\
\hline 5. AS & 56.02 & 13.8 & 0.32 & -0.05 & 0.03 & 0.01 & 0.03 & $-0.23^{* * *}$ & - & \\
\hline 6. LCS & 3.35 & 0.72 & -0.33 & 0.16 & $0.13^{* * *}$ & 0.00 & 0.01 & $0.06^{*}$ & $-0.27^{* * *}$ & - \\
\hline 7. GTS & 3.08 & 0.8 & -0.07 & -0.07 & $0.11^{* * *}$ & $0.14^{* * *}$ & $0.14^{* * *}$ & $0.16^{* * *}$ & $-0.29^{* * *}$ & $0.57^{* * *}$ \\
\hline
\end{tabular}

$N=1752$. Formal, study and class AIMG represent participation in these AIMGs; AP represents academic performance; AS represents academic stress; GTS represents perception of effective teaching; LCS represents the social experience of a learning community

${ }^{\mathrm{a}}$ Less than $40 \%=1 ; 40-45 \%=2 ; 45-50 \%=3 ; 50-55 \%=4 ; 55-60 \%=5 ; 60-65 \%=6 ; 65-70 \%=7 ; 70-75 \%=8$; $75-80 \%=9 ; 80-85 \%=10 ;$ more than $85 \%=11$

*** $p<0.001,{ }^{* *} p<0.01,{ }^{*} p<0.05$ 
Table 3 Results of multiple linear regression predicting academic performance

\begin{tabular}{llllll}
\hline Variable & $B$ & SE & $\beta$ & $t$ & $95 \%$ CI \\
\hline Formal AIMG & -0.07 & 0.02 & -0.10 & $-4.14^{* * *}$ & {$[-0.13,-0.07]$} \\
Class AIMG & 0.01 & 0.01 & 0.02 & 0.98 & {$[0.00,0.05]$} \\
Study AIMG & 0.02 & 0.01 & 0.05 & $2.27^{*}$ & {$[0.04,0.07]$} \\
Academic stress & -0.04 & 0.00 & -0.29 & $-12.217^{* * *}$ & {$[-0.30,-0.28]$} \\
Good teaching & -0.24 & 0.06 & -0.11 & $-3.90^{* * *}$ & {$[-0.23,0.01]$} \\
Learning community & 0.36 & 0.06 & 0.15 & $5.25^{* * *}$ & {$[0.01,0.28]$} \\
$R^{2}$ & 0.21 & & & & \\
adj. R & 0.20 & & & & \\
F Statistic & $20.68^{* * *}(\mathrm{df}=22 ; 1729)$ & & \\
\hline
\end{tabular}

$N=1752$. $B$ represents unstandardised regression coefficients; SE represents the standard error of $B ; \beta$ represents standardised regression coefficients

*** $p<0.001,{ }^{* *} p<0.01,{ }^{*} p<0.05$

$\left(\operatorname{adj} . R^{2}=.20 ; F(22 ; 1729)=20.68, p<.001\right)$, with academic stress as the strongest predictor $(\beta=-.29, p<.001)$. The second strongest predictor is experience of learning community which has a positive effect on academic performance $(\beta=.15, p<.001)$. Formal AIMG participation negatively predicts academic performance $(\beta=-.10, p<.001)$, while study AIMG participation is a positive albeit negligible predictor $(\beta=.05, p<.05)$.

To address RQ4, we conducted a multiple linear regression predicting academic stress with formal, class and study AIMG participation, academic performance, perception of teaching quality, and social experience of learning community, while controlling for demographic variables. Table 4 presents the results of this regression. Overall, the model predicts $25 \%$ of the variance in academic performance (adj. $R^{2}=$ $.25 ; F(22 ; 1729)=27.24, p<.001)$, with academic performance as the strongest predictor $(\beta=-.27, p<.001)$. Additionally, both GTS $(\beta=-.19, p<.001)$ and LCS $(\beta=-.15, p<.001)$ negatively predict academic stress, with participation in study AIMGs as the only positive predictor $(\beta=.08, p<.001)$.

To address RQ5 we conducted a multiple linear regression predicting students' perception of their teaching quality (as presented by GTS) with formal, class and study AIMG participation, academic performance, academic stress, and social experience of learning community, while controlling for demographic variables. Table 5 presents the results of this regression. Overall, the model predicts $40 \%$ of the variance in academic performance (adj. $\left.R^{2}=.40 ; F(22 ; 1729)=54.82, p<.001\right)$, with experience of learning community as the strongest predictor $(\beta=.56, p<.001)$ and formal AIMG participationas the only other positive predictor $(\beta=.07, p<.01)$. Three negative predictors were found in academic stress $(\beta=-.15, p<.001)$, academic performance $(\beta=-.08, p<.001)$ and participation in class AIMGs $(\beta=-.08, p<.001)$. 
Table 4 Results of multiple linear regression predicting academic stress

\begin{tabular}{llllll}
\hline Variable & $B$ & $\mathrm{SE}$ & $\beta$ & $t$ & $95 \% \mathrm{CI}$ \\
\hline Formal AIMG & 0.10 & 0.12 & 0.02 & 0.90 & {$[-0.21,0.25]$} \\
Class AIMG & 0.05 & 0.08 & 0.02 & 0.66 & {$[-0.14,0.17]$} \\
Study AIMG & 0.23 & 0.06 & 0.08 & $3.60^{* * *}$ & {$[-0.04,0.21]$} \\
Academic performance & -2.09 & 0.17 & -0.27 & $-12.21^{* * *}$ & {$[-0.61,0.06]$} \\
Good teaching & -3.24 & 0.46 & -0.19 & $-7.10^{* * *}$ & {$[-1.08,0.71]$} \\
Learning community & -2.85 & 0.51 & -0.15 & $-5.53^{* * *}$ & {$[-1.16,0.86]$} \\
$R^{2}$ & 0.26 & & & & \\
adj. $\mathrm{R}^{2}$ & 0.25 & & & & \\
F Statistic & $27.24^{* * *}(\mathrm{df}=22 ; 1729)$ & & & \\
\hline
\end{tabular}

$N=1752 . B$ represents unstandardised regression coefficients; SE represents the standard error of $B ; \beta$ represents standardised regression coefficients

*** $p<0.001,{ }^{* *} p<0.01,{ }^{*} p<0.05$

Finally, to address RQ 6 we conducted a multiple linear regression predicting students' experience of being part of a learning community with formal, class and study AIMG participation, academic performance, academic stress and GTS, while controlling for demographic variables. Table 6 presents the results of this model. Overall, the model predicts $41 \%$ of variance in the dependent variable (adj. $\left.R^{2}=.41 ; F(22 ; 1729)=57.14, p<.001\right)$, with GTS as the strongest predictor $(\beta=.55, p<.001)$. Both class $(\beta=.10, p<.001)$ and study $(\beta=.08, p<.001)$ AIMG participation predicted experiences of belong to a learning community, while participation in formal AIMGs did not.

Table 5 Results of multiple linear regression predicting students' perception of teaching effectiveness

\begin{tabular}{llllll}
\hline Variable & $B$ & SE & $\beta$ & $t$ & $95 \%$ CI \\
\hline Formal AIMG & 0.02 & 0.01 & 0.07 & $3.29^{* *}$ & {$[0.06,0.08]$} \\
Class AIMG & -0.02 & 0.00 & -0.08 & $-3.94^{* * *}$ & {$[-0.09,-0.08]$} \\
Study AIMG & 0.00 & 0.00 & 0.01 & 0.54 & {$[0.00,0.02]$} \\
Academic performance & -0.04 & 0.01 & -0.08 & $-3.90^{* * *}$ & {$[-0.10,-0.06]$} \\
Academic stress & -0.01 & 0.00 & -0.15 & $-7.09^{* * *}$ & {$[-0.15,-0.15]$} \\
Learning community & 0.63 & 0.02 & 0.56 & $27.94^{* * *}$ & {$[0.52,0.61]$} \\
$R^{2}$ & 0.41 & & & \\
adj. R & 0.40 & $54.82^{* * *}(\mathrm{df}=22 ; 1729)$ & & & \\
F Statistic & & & & \\
\hline
\end{tabular}

$N=1752$. $B$ represents unstandardised regression coefficients; SE represents the standard error of $B ; \beta$ represents standardised regression coefficients

*** $p<0.001,{ }^{* *} p<0.01,{ }^{*} p<0.05$ 
Table 6 Results of multiple linear regression predicting students' social experience of a learning community

\begin{tabular}{llllll}
\hline Variable & $B$ & SE & $\beta$ & $t$ & $95 \% \mathrm{CI}$ \\
\hline Formal AIMG & 0.00 & 0.01 & 0.01 & 0.63 & {$[0.00,0.02]$} \\
Class AIMG & 0.02 & 0.00 & 0.10 & $4.90^{* * *}$ & {$[0.10,0.11]$} \\
Study AIMG & 0.01 & 0.00 & 0.08 & $3.77^{* * *}$ & {$[0.07,0.08]$} \\
Academic performance & 0.04 & 0.01 & 0.11 & $5.25^{* * *}$ & {$[0.09,0.12]$} \\
Academic stress & -0.01 & 0.00 & -0.12 & $-5.529^{* * *}$ & {$[-0.12,-0.11]$} \\
Good teaching & 0.50 & 0.02 & 0.55 & $27.939^{* * *}$ & {$[0.52,0.59]$} \\
$R^{2}$ & 0.42 & & & & \\
adj. $\mathrm{R}^{2}$ & 0.41 & & & & \\
F Statistic & $57.14^{* * *}(\mathrm{df}=22 ; 1729)$ & & & \\
\hline
\end{tabular}

$N=1752 . B$ represents unstandardised regression coefficients; SE represents the standard error of $B ; \beta$ represents standardised regression coefficients

${ }^{* * *} p<0.001,{ }^{* *} p<0.01,{ }^{*} p<0.05$

\section{Discussion}

The present study investigated the adoption, use purposes, perceived outcomes, and role of academic instant messaging groups in academic performance, academic stress and course-related experiences among university students. To this end we conducted a survey-based, exploratory study among a sample of undergraduate university students.

Our data indicate, firstly, that the average student simultaneously participates in a high number of AIMGs. Assuming a student is enrolled in six courses during the semester, the median number of simultaneous AIMGs he/she participates in is nine, of which six are class groups. Secondly, our data confirm the appropriateness of the a priori AIMG typology adopted in this study (see Table 1), with respondents indicating use across all three group types defined. Of the three types, class AIMGs are used in the most courses, followed by study and formal AIMGs. The relatively low use of formal AIMGs should be interpreted in relation to the policies and norms which guide teaching and learning strategy at the institution where the data were collected. The institution's policy prescribes the use of a centralised, web-based learning management system (LMS) as the main platform for the distribution of administrative and learning material and, as our data indicate, formal AIMGs on WhatsApp are only adopted in a relatively small minority of courses. This suggests that, for the most part, academic staff continued to utilise the LMS to communicate with their students during online teaching rather than adopting WhatsApp-based formal AIMGs. It is possible, however, that some academic staff adopted formal AIMGs to augment the LMS, in which case the formal AIMG use levels we report here are higher than what they would be during the institution's normal (face-to-face) class schedules. It is also possible that formal AIMGs may have been initiated on alternative platforms (e.g., Microsoft Teams), in which case they would not be reflected in our data. 
The dearth of previous studies of class AIMGs makes it difficult to gauge whether the high levels of class AIMG participation observed among our sample is unique to their institution, or whether the same pattern would be observable at other universities in the same country or internationally. As shown in Fig. 2, a very small proportion of students frequently post messages or share files in class AIMGs, while a large majority only read. This imbalance between the amount of participants that actively post or share material and those who merely consume content, often referred to as the $1 \%$ rule or $90-9-1$ principle, has been reported in relation to various forms of social media (van Dijck, 2013).

Study AIMGs are notably different in this regard as a larger proportion of students actively participate through posting or sharing. When considering these differences in relation to the use purposes for the different groups (Fig. 3), it suggest that class AIMGs might be used primarily for the distribution of administrative or academic material by students fulfilling either official or unofficial leadership roles in the class (e.g., student representatives). While both class and study AIMGs are used to share notes and discuss academic content, study AIMGs seem to play a particularly important role in group work coordination, which explains the active participation by a larger proportion of users. Additionally, the more frequent use of study AIMGs to share messages of support and encouragement suggests that members of these groups know each other personally. Moreover, study AIMGs are used most frequently to share answers or solutions to assignments, suggesting a certain degree of trust among members. In accordance with Alghamdi et al. (2016), we acknowledge that such sharing of answers may, at times, function as a "cheating technique", but that the online learning setting complicates distinctions between, on the one hand, collaboration through knowledge sharing and, on the other, academic cheating.

In terms of the outcomes students associate with the different group types (see Fig. 4), the high degree of similarity between formal and class AIMGs is noteworthy. It seems, based on these findings, that the two group types, while initiated and managed by different stakeholders, are perceived to serve very similar, primarily administrative functions with emphasis on the distribution of academic material and accurate administrative information. Study AIMGs, by contrast, are perceived as having a stronger impact on learning and academic performance, as well as students' management of academic stress. These findings suggest that students experience these smaller groups to play a more important role in their academic development, while formal and class AIMGs serve functions similar to an online bulletin board with the added affordances of mobility and immediacy.

In contrast to students' perceptions about the value of study AIMGs for their academic performance, consideration of our regression models suggests that participation in AIMGs has little effect. While participation in study AIMGs positively predicts academic performance, the effect, when considering the standardised coefficient values and the relatively low predictive capacity of the model, is negligible. Interestingly, notwithstanding the diminutive magnitude of the effect, our model indicates that formal AIMG participation negatively predicts academic performance, contradicting the findings of earlier studies indicating positive association between AIMG participation and performance in particular courses or assessments (e.g., 
Barhoumi 2015; Nitza and Roman 2016; Avci \& Adiguzel 2017). However, our findings should be distinguished from those made in these studies. Firstly, we considered general academic performance across all courses, not only those in which AIMGs were adopted. Secondly, compared to organic AIMGs, only a small portion of AIMG participation reported by our sample occurred in formal AIMGs. Over and above these aspects, though negative, the size of the effect is too small to be of practical significance within the broader scope of factors that predict academic performance.

The same pattern is observable in relation to academic stress. In contrast to students' perceptions that participation in study AIMGs reduces academic stress, our model (see Table 4) indicates that study AIMG participation positively predicts academic stress, though the effect size is small. This finding may indicate that students experiencing high levels of academic stress participate in study AIMGs as a coping mechanism. However, it may also indicate that study AIMGs exasperate academic stress through the social contagion of stress-related beliefs and perceptions of academic self-efficacy as proposed by Burgess et al. (2018). Our data do not permit causal inference and, as such, these interpretations should be read as propositions which require further investigation. Importantly, across group types, no strong (or even moderate) relationship between participation and increases in reported academic stress was observed. Therefore, while not necessarily reducing stress, as students perceive, participation in AIMGs is not associated with increases in experiences of academic stress in any meaningful way.

Both class and study AIMG participation positively predict students' social experience of being part of a learning community. However, the relative sizes of these effects are small compared to the role of teaching quality which is also a positive predictor. This finding suggests, firstly, that previous studies may have over estimated the role of formal AIMGs as a means of creating a sense of community among students and, secondly, that effective teaching is a much more important factor in this regard than AIMG participation. Given students' perceived outcomes of the important role of study AIMGs in their academic development, it is perhaps surprising that participation in these groups is not a stronger positive predictor of students' experience of a learning community, with both academic performance and academic stress having greater predictive capacity in our model.

Lastly, we consider students' perceptions of teaching effectiveness. Our model again indicates small effects for participation in AIMGs, with formal AIMGs as a positive and class AIMGs as a negative predictor. One may argue that students' participation in formal AIMGs is associated with higher perceptions of the quality of teaching they receive, suggesting that they experience these groups as an effective teaching strategy. This argument, however, is partly contradicted by the lack of effect of formal AIMG participation on academic performance. Hence, while formal AIMGs, much like study AIMGs, may be perceived as effective tools for teaching and learning, our data suggest that they do not impact participants' academic performance. The negative effect of class AIMG participation may be indicative of the role that class AIMGs play in shaping students' perceptions of their courses and lecturers. We expect that messages in class AIMGs, at times, involve criticism of aspects of the course (e.g., content, presentation, assessment etc.) and that such criticism influence participants' subjective evaluations of their courses. These groups, accordingly, run 
the risk of becoming spaces where students share their frustrations, potentially cultivating negative attitudes towards course content and resentment towards teaching staff. These propositions, however, should be considered with respect to the small effect sizes observed and the current lack of qualitative data about the functioning of organic AIMGs.

\subsection{Implications for research and practice}

While our findings provide a useful initial picture of the use and role of AIMGs in higher education, there remains much uncertainty about the phenomenon. We propose, in particular, that qualitative work be conducted to investigate the use patterns of study AIMGs. Of the three group types we considered, it is the one which students perceive as the most valuable for their performance and wellbeing. Specifically, the rather surprising finding that study AIMG use is positively associated with academic stress should be investigated further as it runs contrary to students' perceptions of the role these groups play.

Considering the relevance of our findings for teaching practices at higher education institutions, a number of implications are worth noting. Because the creation and use of organic AIMGs fall outside the locus of control of teaching staff, they have very limited control over the practice or its impacts on teaching and learning. Moreover, we expect that organic AIMGs will remain a popular medium for communication among students in the foreseeable future. While we propose that teaching staff should be mindful of the existence of these groups, our findings suggest that they are not a cause for serious concern in terms of either academic performance or stress. Accordingly, we do not see any reason to actively discourage participation in such groups among students.

Based on our findings we question the value of the adoption of formal AIMGs as part of a teaching strategy. Despite the small negative association we found between formal AIMG participation and academic performance, we are mindful of the implications of such groups for students' expectations of lecturer availability. In rare cases where teaching loads are low and classes are small, effective formal AIMGs may indeed be feasible and effective. However, considering the high levels of work-related stress and burnout among academics (Mudrak et al., 2018), the expectation that lecturers should be available continuously and respond promptly to students through AIMGs is likely to exacerbate the problem. This is not to disregard the importance of a clear communication channel for the dissemination of administrative information from staff to students, such channels should obviously be created and managed.

\subsection{Limitations}

A number of important limitations characterise the present study. Firstly, our measures of AIMG participation are self-developed and non-validated. While we are confident that the data is a meaningful reflection of students' behaviour, we acknowledge that there are may alternative and potentially better ways to quantify AIMG participation. Secondly, the limitations of self-reported data about media use behaviour have been well-documented (Ellis et al., 2019) and we acknowledge the 
possibility of inaccurate findings as a result thereof. Thirdly, as noted in earlier sections, we collected data while teaching at the institution was done online as a result of Covid-19. Our data indicate that, despite this, organic AIMGs were still being used extensively, but we are uncertain of the potential impacts of the switch to online learning on their functioning. Lastly, because we focused specifically on WhatsApp-based AIMGs, our finding are ignorant of AIMGs on alternative platforms. While we are confident that a large majority of AIMGs at the institution are Whatsapp-based, we are mindful of the possibility that students have adopted a variety of online platforms for this purpose, particularly following the switch to online teaching.

\section{Conclusion}

In conclusion, we argue that three primary findings describe the contribution of the present study to the body of knowledge about AIMGs. Firstly, our data indicate that participation in organic AIMGs is an important dimension of academic interaction among university students. These groups have been largely ignored by scholars and their role in the wellbeing and academic development of students has received little attention. The present study is, to the best of our knowledge, a first attempt to provide a comprehensive, quantitative description of organic AIMGs. Secondly, the distinction we propose between study and class AIMGs is of importance to understanding the purposes, outcomes and effects of organic AIMGs. These two groups types are clearly created and used with different goals in mind, and their effects on students' wellbeing and academic experiences are different. While these two types provide a starting point, we propose that a more textured typology of organic AIMGs should be developed to serve as a foundation for studying them in greater depth. Finally, our findings indicate that neither formal nor organic AIMGs play an important role in academic performance, academic stress or course perception among university students. While we did find statistically significant effects for these outcomes, they are not large enough to be considered practically significant. We argue, accordingly, that these groups are, in general, neither particularly useful nor particularly harmful in the context of higher education.

Supplementary Information The online version contains supplementary material available at https://doi.org/10.1007/s10639-021-10631-y.

Funding This research did not receive any specific grant from funding agencies in the public, commercial, or not-for-profit sectors.

Data Availability All data and material is publicly available through the OSF at: https://osf.io/t576r/

Code Availability All code is publicly available through the OSF at: https://osf.io/t576r/

\section{Declarations}

Conflict of Interests The author(s) declare that there are no conflicts of interest with respect to the authorship or the publication of this article. 
Ethics approval Ethical approval for the research was obtained from the relevant committee on Research Ethics at the authors' home institution.

Consent to participate All participants provided informed consent to participate in the research.

Consent for Publication All participants provided consent for publication of the data and research.

\section{References}

Afful, B., \& Akrong, R. (2020). WhatsApp and academic performance among undergraduate students in Ghana: Evidence from the University of Cape Coast. Journal of Education for Business, 95(5), 288296. https://doi.org/10.1080/08832323.2019.1644276.

AlFaris, E., Irfan, F., Ponnamperuma, G., Jamal, A., Vleuten, C. V., Maflehi, N. A., ..., \& Ahmed, A.M.A. (2018). The pattern of social media use and its association with academic performance among medical students. Medical Teacher, 40(sup1), S77-S82. https://doi.org/10.1080/0142159X.2018.1465536.

Alghamdi, E. A., Rajab, H., \& Shah, S.R. (2016). Unmonitored students self-created WhatsApp groups in distance learning environments: A collaborative learning tool or cheating technique. International Journal of Research Studies in Educational Technology, 5(2). https://doi.org/10.5861/ijrset.2016.1604.

Al-Rahmi, W. M., \& Zeki, A. M. (2017). A model of using social media for collaborative learning to enhance learners? performance on learning. Journal of King Saud University - Computer and Information Sciences, 29(4), 526-535. https://doi.org/10.1016/j.jksuci.2016.09.002.

Altaany, F. H. (2015). Usage whatsapp application for e-learning and its impact on academic performance in irbid national university in Jordan. International Journal of Applied Engineering Research, 10(19), 39875-39879.

Avci, H., \& Adiguzel, T. (2017). A case study on mobile-blended collaborative learning in an english as a foreign language (EFL) context. International Review of Research in Open and Distance Learning, 18(7), 45-58. https://doi.org/10.19173/irrodl.v18i7.3261.

Baishya, D., \& Maheshwari, S. (2020). Whatsapp groups in academic context: Exploring the academic uses of whatsapp groups among the students. Contemporary Educational Technology, 11(1), 31-46. https://doi.org/10.30935/cet.641765.

Barhoumi, C. (2015). The effectiveness of WhatsApp mobile learning activities guided by activity theory on students' knowledge management. Contemporary Educational Technology, 6(3), 221-238.

Basitere, M., \& Ivala, E. N. (2017). An exploration of students' experiences of Blended Learning in a physics course at a university of technology. Journal of Social Development in Africa, 32(1), $23-43$.

Basitere, M., Mogashana, D., \& Ivala, E.N. (2019). The influence of whatsapp social media on students? learning and performance in engineering mathematics at the extended curriculum programme. In ECSM 2019 6th European conference on social media (p. 23).

Bouhnik, D., \& Deshen, M. (2014). WhatsApp goes to school: mobile instant messaging between teachers and students. Journal of Information Technology Education: Research, 13, 217-231. https://doi.org/10.28945/2051.

Bowman, N. D., \& Keene, J. R. (2018). A layered framework for considering open science practices. Communication Research Reports, 35(4), 363-372. https://doi.org/10.1080/08824096.2018.1513273.

Burgess, L. G., Riddell, P. M., Fancourt, A., \& Murayama, K. (2018). The influence of social contagion within education: a motivational perspective. Mind, Brain, and Education, 12(4), 164-174. https://doi.org/10.1111/mbe.12178.

Dienlin, T., Johannes, N., Bowman, N. D., Masur, P. K., Engesser, S., Kümpel, A. S., ..., \& de Vreese, C. (2020). An agenda for open science in communication. Journal of Communication, jqz052. https://doi.org/10.1093/joc/jqz052.

Ellis, D. A., Davidson, B. I., Shaw, H., \& Geyer, K. (2019). Do smartphone usage scales predict behavior?. International Journal of Human-Computer Studies, 130, 86-92. https://doi.org/10.1016/j.ijhcs.2019.05.004. 
Fuentes Gutiérrez, V., Garća Domingo, M., \& Aranda López, M. (2017). Groups of class; Groups of WhatsApp. Analysis of communicative dynamics among university students. Prisma Social, 18, 144 171.

Griffin, P., Coates, H., Mcinnis, C., \& James, R. (2003). The development of an extended course experience questionnaire. Quality in Higher Education, 9(3), 259-266. https://doi.org/10.1080/135383203200015111.

Hickerson, C. A., \& Giglio, M. (2009). Instant messaging between students and faculty: a tool for increasing student-faculty interaction. International Journal on E-Learning, 8(1), 71-88. (Publisher: Association for the Advancement of Computing in Education (AACE)).

Javaheri, A. (2017). Psychological capital: An internal resource for counseling students coping with academic and clinical stress. Unpublished doctoral dissertation, The College of William and Mary in Virginia. https://doi.org/10.21220/W4DT0B.

Kim, H., Lee, M., \& Kim, M. (2014). Effects of mobile instant messaging on collaborative learning processes and outcomes: the case of South Korea. Journal of Educational Technology \& Society, 17(2), 13.

Krause, H.-V., Baum, K., Baumann, A., \& Krasnova, H. (2019). Unifying the detrimental and beneficial effects of social network site use on self-esteem: a systematic literature review. Media Psychology, 0(0), 1-38. https://doi.org/10.1080/15213269.2019.1656646.

Lakaev, N. (2009). Validation of an Australian academic stress questionnaire. Australian Journal of Guidance and Counselling, 19(1), 56-70. https://doi.org/10.1375/ajgc.19.1.56.

le Roux, D. B., \& Parry, D. A. (2021). Off-task media use in academic settings: cycles of self-regulation failure. Journal of American College Health, 69(2), 134-141. https://doi.org/10.1080/07448481.2019.1656636.

le Roux, D. B., \& Parry, D. A. (2020). The town square in your pocket: exploring four metaphors of social media. In M. Hattingh, M. Matthee, H. Smuts, I. Pappas, Y. K. Dwivedi, \& M. Mäntymäki (Eds.) Responsible design, implementation and use of information and communication technology. Lecture Notes in Computer Science (pp. 187-198). Cham: Springer International Publishing.

le Roux, D. B., Parry, D. A., Totolo, A., Iyawa, G., Holloway, J., Prenter, A., \& Botha, L. (2021). Media multitasking, online vigilance and academic performance among students in three Southern African countries. Computers \& Education, 160, 104056. https://doi.org/10.1016/j.compedu.2020.104056.

Leung, L. (2001). College student motives for chatting on ICQ. New Media \& Society, 3(4), 483-500. https://doi.org/10.1177/14614440122226209, Publisher: SAGE Publications.

Lewis, N. A. (2020). Open communication science: A primer on why and some recommendations for how. Communication Methods and Measures, 14(2), 71-82.

Macgeorge, E. L., Samter, W., \& Gillihan, S.J. (2005). Academic stress, supportive communication, and health a version of this paper was presented at the 2005 international communication association convention in New York City. Communication Education, 54(4), 365-372. https://doi.org/10.1080/03634520500442236.

Mcinnis, C., Griffin, P., James, R., \& Coates, H. (2001). Development of the Course Experience Questionnaire (CEQ)(Tech. Rep.). Melbourne: Department of Education, Training and Youth Affairs.

Mogashana, D., Basitere, M., Ivala, E. N., \& Mapatagane, N. (2019). The use of whatsapp social media for providing psychosocial support to first-year chemical engineering extended curriculum programme students. In ECSM 2019 6th European conference on social media (pp. 208-214).

Mudrak, J., Zabrodska, K., Kveton, P., Jelinek, M., Blatny, M., Solcova, I., \& Machovcova, K. (2018). Occupational well-being among university faculty: A job demands-resources model. Research in Higher Education, 59(3), 325-348. https://doi.org/10.1007/s11162-017-9467-x.

Muktar, B., Sagagi, F. M., Gumel, M. F., \& Hussein, A. (2020). An application of self-regulated learning theory in assessing effect of ICT mediated academic communication amongst Federal University dutse undergraduate students. JCCR - Journal of Community \& Communication Research, 5(2), 139-143.

Nitza, D., \& Roman, Y. (2016). WhatsApp messaging: achievements and success in academia. International Journal of Higher Education, 5(4), 255. https://doi.org/10.5430/ijhe.v5n4p255.

Ntinda, M. N., \& Bidwell, N. J. (2018). Solo or peers: Technology mediated learning of programming. In Proceedings of the second African conference for human computer interaction: Thriving communities (pp. 192-195). 
Orben, A. (2020). Teenagers, screens and social media: a narrative review of reviews and key studies. Social Psychiatry and Psychiatric Epidemiology, 55(4), 407-414. https://doi.org/10.1007/s00127-019-01825-4.

Panova, T., Carbonell, X., Chamarro, A., \& Puerta-Cortés, D.X. (2020). Specific smartphone uses and how they relate to anxiety and depression in university students: a cross-cultural perspective. Behaviour \& Information Technology, 39(9), 944-956. https://doi.org/10.1080/0144929X.2019.1633405.

Parr, J. M., \& Townsend, M. A. R. (2002). Environments, processes, and mechanisms in peer learning. International Journal of Educational Research, 37(5), 403-423. https://doi.org/10.1016/S0883-0355(03)00013-2.

Poblete, P., \& Nieto, E. (2020). Does time matter? WhatsApp vs electronic mail for dental education. A pilot study. European Journal of Dental Education, 24(1), 121-125. https://doi.org/10.1111/eje.12475.

Pérez-Jorge, D., Barragán-Medero, F., Gutiérrez-Barroso, J., \& Castro-León, F (2018). A synchronous tool for innovation and improvement of university communication, counseling and tutoring: The WhatsApp experience. EURASIA Journal of Mathematics, Science and Technology Education, 14(7). https://doi.org/10.29333/ejmste/90588.

Quan-Haase, A., \& Young, A. L. (2010). Uses and gratifications of social media: a comparison of Facebook and instant messaging. Bulletin of Science, Technology \& Society, 30(5), 350-361. Publisher: SAGE Publications Inc.

R. Core Team (2020). R: A language and environment for statistical computing [Computer software manual]. Vienna, Austria. Retrieved from https://www.R-project.org/.

Rahmadi, I. (2020). Whatsapp group for teaching and learning in indonesian higher education what's up?. International Journal of Interactive Mobile Technologies, 14(13), 150-160. https://doi.org/10.3991/ijim.v14i13.14121.

Román, P., Ballesteros, C., \& Noguera, M. (2018). Academic whatsapp groups as alternative communication and motivation systems in higher education. Revista Espacios, 39(10).

Suardika, I. K., Suhartini, L., \& Pasassung, N. (2020). Using whatsapp for teaching a course on the education profession: Presence, community and learning. International Journal of Mobile and Blended Learning (IJMBL), 12(1), 17-32. https://doi.org/10.4018/IJMBL.2020010102.

Udenze, S., \& Oshionebo, B. (2020). Investigating ?Whatsapp? for collaborative learning among undergraduates. Üsküdar Üniversitesi İletişim Fakültesi Akademik Dergisi Etkileşim, 5, 24-50.

van Dijck, J. (2013). The culture of connectivity: a critical history of social media. Oxford: Oxford University Press.

Vorderer, P., Hefner, D., Reinecke, L., Klimmt, C., Hefner, D., Reinecke, L., \& Klimmt, C. (2017). Permanently online, permanently connected : Living and communicating in a POPC world. Evanston: Routledge.

WhatsApp (2020). About WhatsApp. https://www.whatsapp.com/about/.

Whiting, A., \& Williams, D. (2013). Why people use social media: a uses and gratifications approach. Qualitative Market Research: An International Journal, 16(4), 362-369. https://doi.org/10.1108/QMR-06-2013-0041, Publisher: Emerald Group Publishing Limited.

Willemse, J. J. (2015). Undergraduate nurses reflections on Whatsapp use in improving primary health care education. Curationis, 38(2), 1512. https://doi.org/10.4102/curationis.v38i2.1512.

Zulkanain, N. A., Miskon, S., \& Syed Abdullah, N. (2020). An adapted pedagogical framework in utilizing WhatsApp for learning purpose. Education and Information Technologies, 25(4), 2811-2822. https://doi.org/10.1007/s10639-019-10096-0.

Publisher's note Springer Nature remains neutral with regard to jurisdictional claims in published maps and institutional affiliations. 